\title{
Reeb-Thurston stability for symplectic foliations
}

\author{
Marius Crainic • Ioan Mărcuţ
}

Received: 10 June 2014 / Revised: 2 December 2014 / Published online: 10 January 2015

C The Author(s) 2015. This article is published with open access at Springerlink.com

\begin{abstract}
We prove a version of the local Reeb-Thurston stability theorem for symplectic foliations.
\end{abstract}

\section{Introduction}

A symplectic foliation on a manifold $M$ is a (regular) foliation $\mathcal{F}$ endowed with a 2-form $\omega$ on $T \mathcal{F}$ whose restriction to each leaf $S$ of $\mathcal{F}$ is a symplectic form

$$
\omega_{S} \in \Omega^{2}(S)
$$

Equivalently, a symplectic foliation is a Poisson structure of constant rank.

In this paper we prove a normal form theorem for symplectic foliations around embedded leaves. The result uses the cohomological variation of $\omega$ at the leaf $S$, which is a linear map (see Sect. 1 for the definition)

$$
\left[\delta_{S} \omega\right]_{x}: v_{x}^{*} \longrightarrow H^{2}\left(\widetilde{S}_{h o l}\right), \quad x \in S
$$

where $v$ denotes the normal bundle of $T \mathcal{F}$, and $\widetilde{S}_{\text {hol }}$ is the holonomy cover of $S$. The cohomological variation arises in fact from a linear map:

$$
\delta_{S} \omega_{x}: v_{x}^{*} \longrightarrow \Omega_{\text {closed }}^{2}\left(\widetilde{S}_{\text {hol }}\right) .
$$

\footnotetext{
M. Crainic

Department of Mathmetics, Utrecht University, 3508 TA Utrecht, The Netherlands e-mail: m.crainic@uu.nl

I. Mărcuţ ( $\bowtie)$

IMAPP, Radboud University Nijmegen, 6500 GL Nijmegen, The Netherlands e-mail: i.marcut@math.ru.nl
} 
The local model for the foliation around $S$, which appears in the classical results of Reeb and Thurston, is the flat bundle $\left(\widetilde{S}_{h o l} \times v_{x}\right) / \pi_{1}(S, x)$, where $\pi_{1}(S, x)$ acts on the second factor via the linear holonomy

$$
d h: \pi_{1}(S, x) \longrightarrow G l\left(v_{x}\right) .
$$

The flat bundle can be endowed with leafwise closed 2-forms, which are symplectic in a neighborhood of $S$; namely, the leaf through $v \in v_{x}$ carries the closed 2-form $j_{S}^{1}(\omega)_{v}$ whose pull-back to $\widetilde{S}_{h o l} \times\{v\}$ is

$$
p^{*}\left(j_{S}^{1}(\omega)_{v}\right)=p^{*}\left(\omega_{S}\right)+\delta_{S} \omega_{x}(v) .
$$

Our main result is the following:

Theorem 1 Let $S$ be an embedded leaf of the symplectic foliation $(M, \mathcal{F}, \omega)$. If the holonomy group of $S$ is finite and the cohomological variation (1) at $S$ is a surjective map, then an open neighborhood of $S$ is isomorphic as a symplectic foliation to an open neighborhood of $S$ in the flat bundle $\left(\widetilde{S}_{\text {hol }} \times v_{x}\right) / \pi_{1}(S, x)$ endowed with the family of closed 2-forms $j_{S}^{1}(\omega)$ by a diffeomorphism which fixes $S$.

This result is not a first order normal form theorem, since the holonomy group and the holonomy cover depend on the germ of the foliation around the leaf. The first order jet of the foliation at $S$ sees only the linear holonomy group $H_{\text {lin }}$ (i.e. the image of $d h$ ) and the corresponding linear holonomy cover denoted by $\widetilde{S}_{l i n}$. Now, the map (2) is in fact the pull-back of a map with values in $\Omega_{\text {closed }}^{2}\left(\widetilde{S}_{l i n}\right)$. This remark, an extension to noncompact leaves of a result of Thurston (Lemma 2), and Theorem 1 imply the following first order normal form result:

Corollary 1 Under the assumptions that $S$ is embedded, $\pi_{1}(S, x)$ is finitely generated, $H_{\text {lin }}$ is finite, $H^{1}\left(\widetilde{S}_{\text {lin }}\right)=0$ and the cohomological variation

$$
\left[\delta_{S} \omega\right]_{x}: v_{x}^{*} \longrightarrow H^{2}\left(\widetilde{S}_{l i n}\right)
$$

is surjective, the conclusion of Theorem 1 holds.

Related phenomena

Our result is clearly related to the normal form theorem for Poisson manifolds around symplectic leaves from [4]. Both results have the same conclusion, yet the conditions of Theorem 1 are substantially weaker. More precisely, for regular Poisson manifolds, the hypotheses of the main result in loc.cit. are (see Corollary 4.1.22 and Lemma 4.1.23 [7]):

- the leaf $S$ is compact,

- $\pi_{1}(S, x)$ is finite,

- the cohomological variation is an isomorphism, when viewed as a map

$$
\left[\delta_{S} \omega\right]_{x}: v_{x}^{*} \longrightarrow H^{2}\left(\widetilde{S}_{\text {uni }}\right),
$$

where $\widetilde{S}_{u n i}$ is the universal cover of $S$. 
There is yet another essential difference between Theorem 1 and the result from [4], namely, even in the setting of Corollary 1, the results presented here have first order assumptions only in the world of symplectic foliations, and not in that of Poisson structures. The information that a Poisson bivector has constant rank is not detectable from its first jet. In fact, the conditions of Theorem 1 allow for Poisson-deformations which increase the rank. To explain this phenomenon, let us consider the simplified setting:

- $S$ is compact and simply connected.

Then, by Corollary 2.7 in [8], the local normal form result from [4] holds under the weaker assumptions:

- $\left[\delta_{S} \omega\right]_{x}$ is surjective, and its kernel is at most one-dimensional.

These conditions have an interpretation in the context of stability of symplectic leaves, from [3]. Namely, for $S$ as above (i.e. compact and simply connected), we have an isomorphism (see the proof of Lemma 2.6 in [8])

$$
H_{\pi, S}^{2}(M) \cong \operatorname{coker}\left(\left[\delta_{S} \omega\right]_{x}\right) \oplus \bigwedge^{2} \operatorname{ker}\left(\left[\delta_{S} \omega\right]_{x}\right),
$$

where $H_{\pi, S}^{\bullet}(M)$ denotes the Poisson cohomology restricted to $S$ (see [3]); thus the conditions are equivalent to $H_{\pi, S}^{2}(M)=0$. By Theorem $2.2[3], H_{\pi, S}^{2}(M)=0$ implies that $S$ is strongly stable, i.e. any Poisson structure close to $\pi$ has a leaf symplectomorphic to $\left(S, \omega_{S}\right)$. Now, the hypotheses of Theorem 1 take care of the first component in (4). The second component controls deformations which change the rank of the Poisson structure. Concretely, $v, w \in \operatorname{ker}\left(\left[\delta_{S} \omega\right]_{x}\right)$ are linearly independent, and $\pi_{\operatorname{lin}, S}$ is the linearized Poisson structure at $S$, then we can interpret $\pi_{\operatorname{lin}, S}+\epsilon v \wedge w, \epsilon>0$, as a deformation which increases the rank by two (see the proof of Theorem 2.3 in [3]). Of course, we do not allow such deformations in the context of symplectic foliations.

Motivated by a question of the referee, we included in the end of the paper some remarks on global aspects of symplectic foliations with surjective cohomological variation.

A weaker version of Theorem 1, in which the leaf is assumed to be of finite type, is part of the $\mathrm{PhD}$ thesis [7] of the second author.

\section{The local model and the cohomological variation}

In this section we describe the local model of a symplectic foliation around a leaf, and introduce the cohomological variation of the symplectic structures. In the case of general Poisson manifolds, the local model was first constructed by Vorobjev [12]. The approach presented here is more direct; for the relation between these two constructions see [7].

Let $(M, \mathcal{F})$ be a foliated manifold, and denote its normal bundle by

$$
v:=T M / T \mathcal{F}
$$


Then $v$ carries a flat $T \mathcal{F}$ connection, called the Bott connection, given by

$$
\nabla: \Gamma(T \mathcal{F}) \times \Gamma(v) \longrightarrow \Gamma(v), \quad \nabla_{X}(\bar{Y}):=\overline{[X, Y]}
$$

where, for a vector field $Z$, we denote by $\bar{Z}$ its class in $\Gamma(v)$. For a path $\gamma$ inside a leaf $S$, parallel transport with respect to $\nabla$ gives the linear holonomy transformations:

$$
d h(\gamma): v_{\gamma(0)} \stackrel{\sim}{\rightarrow} v_{\gamma(1)}
$$

This map depends only on $\gamma$ modulo homotopies inside $S$ with fixed endpoints. Applying $d h$ to closed loops at $x$, we obtain the linear holonomy group

$$
H_{l i n, x}:=d h\left(\pi_{1}(S, x)\right) \subset G l\left(v_{x}\right) .
$$

The linear holonomy cover of a leaf $S$ at $x$, denoted by $\widetilde{S}_{l i n, x}$ is the covering space corresponding to the kernel of $d h$; thus it is a principal $H_{l i n, x}$ bundle over $S$. Also, $\widetilde{S}_{l i n, x}$ can be defined as the space of classes of paths in $S$ starting at $x$, where we identify two such paths if they have the same endpoint and they induce the same holonomy transport.

The Bott connection induces a foliation $\mathcal{F}_{v}$ on $v$ whose leaves are the orbits of $d h$; i.e. the leaf of $\mathcal{F}_{v}$ through $v \in v_{x}$ covers the leaf $S$ through $x$, and is given by

$$
\widetilde{S}_{v}:=\{d h(\gamma) v: \gamma \text { is a path in } S \text { starting at } x\}
$$

Therefore, $\widetilde{S}_{l i n, x}$ covers of the leaves of the foliation $\mathcal{F}_{v}$ above $S$ via the maps

$$
p_{v}: \widetilde{S}_{l i n, x} \longrightarrow \widetilde{S}_{v}, \quad p_{v}([\gamma])=d h(\gamma) v, \quad v \in v_{x} .
$$

The local model of the foliation around the leaf $S$ is the foliated manifold

$$
\left(v_{S}, \mathcal{F}_{v_{S}}\right), \quad \text { where } \mathcal{F}_{v_{S}}:=\left.\mathcal{F}_{v}\right|_{v_{S}}
$$

The linear holonomy induces an isomorphism between the local model and the flat bundle from the Introduction

$$
\left(\widetilde{S}_{l i n, x} \times v_{x}\right) / H_{l i n, x} \stackrel{\sim}{\rightarrow} v_{S}, \quad[\gamma, v] \mapsto p_{v}([\gamma]) .
$$

Consider now a symplectic structure $\omega$ on the foliation $\mathcal{F}$, i.e. a 2-form on $T \mathcal{F}$

$$
\omega \in \Omega^{2}(T \mathcal{F})
$$

whose restriction to each leaf is symplectic. We first construct a closed foliated 2-form $\delta \omega$ on $\left(v, \mathcal{F}_{v}\right)$, which represents the derivative of $\omega$ in the transversal direction. For this, choose an extension $\widetilde{\omega} \in \Omega^{2}(M)$ of $\omega$ and let

$$
\Omega(X, Y):=d \widetilde{\omega}(X, Y, \cdot), \quad X, Y \in T \mathcal{F}
$$

Since $\omega$ is closed along the leaves of $\mathcal{F}, \Omega(X, Y) \in v^{*}$, thus $\Omega \in \Omega^{2}\left(T \mathcal{F} ; v^{*}\right)$. 
Now, the dual of the Bott connection on $v^{*}$ induces a differential $d \nabla$ on the space of foliated forms with values in the conormal bundle $\Omega^{\bullet}\left(T \mathcal{F} ; v^{*}\right)$; this can be given explicitly by the classical Koszul formula

$$
\begin{aligned}
& d_{\nabla}: \Omega^{\bullet}\left(T \mathcal{F} ; v^{*}\right) \longrightarrow \Omega^{\bullet+1}\left(T \mathcal{F} ; v^{*}\right), \\
d_{\nabla} \eta\left(X_{0}, \ldots, X_{p}\right)= & \sum_{i}(-1)^{i} \nabla_{X_{i}} \eta\left(X_{0}, \ldots, \widehat{X}_{i}, \ldots, X_{p}\right)+ \\
& +\sum_{i<j}(-1)^{i+j} \eta\left(\left[X_{i}, X_{j}\right], X_{0}, \ldots, \widehat{X}_{i}, \ldots, \widehat{X}_{j}, \ldots, X_{p}\right),
\end{aligned}
$$

for $\eta \in \Omega^{p}\left(T \mathcal{F} ; v^{*}\right), X_{i} \in \Gamma(T \mathcal{F})$. Denote the resulting cohomology by $H^{\bullet}\left(\mathcal{F} ; v^{*}\right)$.

It is easy to see that $\Omega$ is $d_{\nabla}$-closed. In fact, this construction can be preformed in all degrees, and it produces a canonical map (see e.g. [2])

$$
d_{v}: H^{\bullet}(\mathcal{F}) \longrightarrow H^{\bullet}\left(\mathcal{F} ; v^{*}\right)
$$

which maps $[\omega]$ to [ $\Omega]$. Also, if $\widetilde{\omega}+\alpha$ is a second extension of $\omega$ (where $\alpha$ vanishes along $\mathcal{F}$ ), then $\Omega$ changes by $d_{\nabla} \lambda$, where $\lambda \in \Omega^{1}\left(T \mathcal{F} ; v^{*}\right)$, is given by

$$
\lambda(X):=\iota_{X} \alpha \text { for } \quad X \in T \mathcal{F} .
$$

Note that there is a natural embedding

$$
\mathcal{J}: \Omega^{\bullet}\left(T \mathcal{F} ; v^{*}\right) \longrightarrow \Omega^{\bullet}\left(T \mathcal{F}_{v}\right), \quad \mathcal{J}(\eta)_{v}:=\left.p^{*}(\langle\eta, v\rangle)\right|_{T \mathcal{F}_{v}}, \quad v \in v,
$$

where $p: v \rightarrow M$ is the projection. It is easy to see that under $\mathcal{J}$ the differential $d_{\nabla}$ corresponds to the leafwise de Rham differential $d_{\mathcal{F}_{v}}$ on the leaves of $\mathcal{F}_{\nu}$. In particular, we obtain a closed foliated 2-form

$$
\delta \omega:=\mathcal{J}(\Omega) \in \Omega^{2}\left(T \mathcal{F}_{v}\right)
$$

which we call the vertical derivative of $\omega$. Since $\delta \omega$ vanishes on $M$ (viewed as the zero section), it follows that $p^{*}(\omega)+\delta \omega$ is nondegenerate on the leaves in an open neighborhood of $M$; thus

$$
\left(\nu, \mathcal{F}_{\nu}, p^{*}(\omega)+\delta \omega\right)
$$

is a symplectic foliation around $M$.

Consider now a symplectic leaf $S$. Restricting $p^{*}(\omega)+\delta \omega$ to the leaves above $S$, we obtain closed foliated 2 -forms along the leaves of the $\mathcal{F}_{v_{S}}$, denoted by

$$
j_{S}^{1}(\omega):=p^{*}\left(\omega_{S}\right)+\delta_{S} \omega \in \Omega^{2}\left(T \mathcal{F}_{\nu_{S}}\right),
$$


where $\omega_{S}:=\left.\omega\right|_{S}$ and $\delta_{S} \omega:=\left.\delta \omega\right|_{\nu_{S}}$. Any open neighborhood of $S$ in

$$
\left(v_{S}, \mathcal{F}_{v_{S}}, j_{S}^{1}(\omega)\right)
$$

on which $j_{S}^{1}(\omega)$ is symplectic will be regarded as the local model of the symplectic foliation around $S$; i.e. we think about the local model as a germ of a symplectic foliation around $S$.

In order to define the cohomological variation of $\omega$, consider first the linear map

$$
\delta_{S} \omega_{x}: v_{x} \longrightarrow \Omega_{\text {closed }}^{2}\left(\widetilde{S}_{\text {lin }, x}\right), \quad v \mapsto p_{v}^{*}\left(\delta_{S} \omega\right)
$$

where the map $p_{v}$ is the covering map defined by (5). By the discussion above, choosing a different extension of $\omega$ changes $p_{v}^{*}\left(\delta_{S} \omega\right)$ by an exact 2-form; hence the cohomology class $\left[p_{v}^{*}\left(\delta_{S} \omega\right)\right]$ is independent of the 2-form $\Omega$ used to construct $\delta_{S} \omega$. The induced linear map to the cohomology of $\widetilde{S}_{\text {lin, } x}$, will be called the cohomological variation of $\omega$ at $S$

$$
\left[\delta_{S} \omega\right]_{x}: v_{x} \longrightarrow H^{2}\left(\widetilde{S}_{l i n, x}\right), \quad v \mapsto\left[p_{v}^{*}\left(\delta_{S} \omega\right)\right]
$$

In the Introduction we denoted the lifts of $\left[\delta_{S} \omega_{x}\right]$ to the holonomy cover $\widetilde{S}_{h o l}$, respectively to the universal cover $\widetilde{S}_{\text {uni }}$ of $S$, by the same symbol.

Up to isomorphism, the local model is independent of the choices involved.

Proposition 1 Different choices of $\Omega \in \Omega^{2}\left(T \mathcal{F}, v^{*}\right)$ satisfying $d_{v}[\omega]=[\Omega]$ produce local models that are isomorphic around $S$ by a diffeomorphism that fixes $S$.

The proof of this result is deferred until the end of next section, because it uses Lemma 5 , which is a foliated version of the Moser Lemma.

\section{Five lemmas}

In this section we prove some auxiliary results used in the proof of Theorem 1.

\section{Reeb Stability around noncompact leaves}

Consider a foliated manifold $(M, \mathcal{F})$ and let $S$ be an embedded leaf. The classical Reeb Stability Theorem (see e.g. [9]) says that, if the holonomy group $H_{\text {hol }}$ is finite and $S$ is compact, then a saturated neighborhood of $S$ in $M$ is isomorphic as a foliated manifold to the flat bundle

$$
\left(\widetilde{S}_{h o l} \times T\right) / H_{h o l},
$$

where $T$ is a small transversal that is invariant under the holonomy action of $\mathrm{H}_{\mathrm{hol}}$. Since actions of finite groups can be linearized, it follows that the holonomy of $S$ equals the linear holonomy of $S$. So, some neighborhood of $S$ in $(M, \mathcal{F})$ is isomorphic as a foliated manifold with the flat bundle from the previous section

$$
\left(\widetilde{S}_{l i n} \times v_{x}\right) / H_{l i n} .
$$


Generalizations to noncompact leaves of the Reeb stability theorem were studied before (see $[1,6]$ and the references therein). The goal in these works is to produce saturated neighborhoods of embedded leaves (also called proper leaves in foliation literature) which are isomorphic to the flat bundle (7). For this, finiteness of the holonomy group is not sufficient [6]. Results are known to hold only in low dimensions [1], for compact ambient manifolds, and with extra restrictions on the topology of the leaf. Below, we show that the direct generalization of the Reeb Stability Theorem for noncompact leaves does work, but at the expense of giving up on the conclusion that the neighborhood be saturated. The proof is a careful adaptation to the noncompact case of the proof of the classical result from [9].

Lemma 1 Let $(M, \mathcal{F})$ be a foliation and let $S \subset M$ be an embedded leaf. If $S$ has finite holonomy, then an open neighborhood of $S$ in $M$ is isomorphic as a foliated space to an open neighborhood of $S$ in the local model (7), by a diffeomorphism which fixes $S$.

Proof Since the holonomy is finite, it equals the linear holonomy, and we denote $H:=H_{h o l}=H_{l i n}$ and $\widetilde{S}:=\widetilde{S}_{h o l}=\widetilde{S}_{l i n}$.

The assumption that $S$ be embedded allows us to restrict to a tubular neighborhood; so we assume that the foliation is on a vector bundle $p: E \rightarrow S$ (with $E \cong v_{S}$ ), for which $S$, identified with the zero section, is a leaf. Then the holonomy of paths in $S$ is represented by germs of a diffeomorphism between the fibers of $E$.

Each point in $S$ has an open neighborhood $U \subset E$ satisfying

- $S \cap U$ is 1-connected,

- for $x \in S \cap U, E_{x} \cap U$ is a connected neighborhood of $x$,

- for every $x, y \in S \cap U$, the holonomy along any path in $S \cap U$ connecting them is defined as a diffeomorphism between the spaces

$$
h_{x}^{y}: E_{x} \cap U \stackrel{\sim}{\rightarrow} E_{y} \cap U .
$$

Let $\mathfrak{U}$ be locally finite cover of $S$ by open sets $U \subset E$ of the type just described, such that for all $U, U^{\prime} \in \mathfrak{U}, U \cap U^{\prime} \cap S$ is connected (or empty), and such that each $U \in \mathfrak{U}$ is relatively compact.

We fix $x_{0} \in S, U_{0} \in \mathfrak{U}$ an open set containing $x_{0}$, and denote by

$$
V:=E_{x_{0}}
$$

Consider a path $\gamma$ in $S$ starting at $x_{0}$ and with endpoint $x$. Cover the path by a chain of open sets in $\mathfrak{U}$

$$
\xi=\left(U_{0}, \ldots, U_{k(\xi)}\right)
$$

such that there is a partition

$$
0=t_{0}<t_{1}<\cdots t_{k-1}<t_{k}=1,
$$


with $\gamma\left(\left[t_{j-1}, t_{j}\right]\right) \subset U_{j}$. Since the holonomy transformations inside $U_{j}$ are all trivial, and all the intersections $U_{i} \cap U_{j} \cap S$ are connected, it follows that the holonomy of $\gamma$ only depends on the chain $\xi$ and is defined as an embedding

$$
h(\gamma)=h_{x_{0}}^{x}(\xi): O(\xi) \hookrightarrow E_{x},
$$

where $O(\xi) \subset V$ is an open neighborhood of $x_{0}$, which is independent of $x \in U_{k(\xi)}$. Denote by $\mathcal{Z}$ the space of all chains in $\mathfrak{U}$

$$
\xi=\left(U_{0}, \ldots, U_{k(\xi)}\right), \text { with } U_{l} \cap U_{l+1} \neq \emptyset .
$$

Denote by $K$ the kernel of $\pi_{1}\left(S, x_{0}\right) \rightarrow H$. The holonomy cover $\widetilde{S} \rightarrow S$ can be described as the space of all paths $\gamma$ in $S$ starting at $x_{0}$, and two such paths $\gamma_{1}$ and $\gamma_{2}$ are equivalent if they have the same endpoint, and the homotopy class of $\gamma_{2}^{-1} \circ \gamma_{1}$ lies in $K$. The projection is then given by $[\gamma] \mapsto \gamma(1)$. Denote by $\widetilde{x}_{0}$ the point in $\widetilde{S}$ corresponding to the constant path at $x_{0}$. So, we can represent each point in $\widetilde{S}$ (not uniquely!) by a pair ( $\xi, x)$ with $\xi \in \mathcal{Z}$ and endpoint $x \in U_{k(\xi)} \cap S$.

The group $H$ acts freely on $\widetilde{S}$ by pre-composing paths. For every $g \in H$ fix a chain $\xi_{g} \in \mathcal{Z}$, such that $\left(\xi_{g}, x_{0}\right)$ represents $\widetilde{x}_{0} g$. Consider the open set

$$
\widetilde{O}_{0}:=\bigcap_{g \in H} O\left(\xi_{g}\right) \subset V,
$$

on which all holonomies $h_{x_{0}}^{x_{0}}\left(\xi_{g}\right)$ are defined, and a smaller open set $\widetilde{O}_{1} \subset \widetilde{O}_{0}$ around $x_{0}$, such that $h_{x_{0}}^{x_{0}}\left(\xi_{g}\right)$ maps $\widetilde{O}_{1}$ into $\widetilde{O}_{0}$. Hence the composition

$$
h_{x_{0}}^{x_{0}}\left(\xi_{g}\right) \circ h_{x_{0}}^{x_{0}}\left(\xi_{h}\right): \widetilde{O}_{1} \hookrightarrow V
$$

is well defined. Since the germs of $h_{x_{0}}^{x_{0}}\left(\xi_{g}\right) \circ h_{x_{0}}^{x_{0}}\left(\xi_{h}\right)$ and $h_{x_{0}}^{x_{0}}\left(\xi_{g h}\right)$ are the same, by shrinking $\widetilde{O}_{1}$ if necessary, we may assume that

$$
h_{x_{0}}^{x_{0}}\left(\xi_{g}\right) \circ h_{x_{0}}^{x_{0}}\left(\xi_{h}\right)=h_{x_{0}}^{x_{0}}\left(\xi_{g h}\right): \widetilde{O}_{1} \hookrightarrow V, \quad \forall g, h \in H .
$$

Consider the following open set

$$
O:=\bigcap_{g \in H} h_{x_{0}}^{x_{0}}\left(\xi_{g}\right)\left(\widetilde{O}_{1}\right)
$$

Then $O \subset \widetilde{O}_{1}$, and for $h \in H$, we have that

$$
\begin{aligned}
h_{x_{0}}^{x_{0}}\left(\xi_{h}\right)(O) & \subseteq \bigcap_{g \in H} h_{x_{0}}^{x_{0}}\left(\xi_{h}\right) \circ h_{x_{0}}^{x_{0}}\left(\xi_{g}\right)\left(\widetilde{O}_{1}\right) \\
& =\bigcap_{g \in H} h_{x_{0}}^{x_{0}}\left(\xi_{h g}\right)\left(\widetilde{O}_{1}\right)=\bigcap_{g \in H} h_{x_{0}}^{x_{0}}\left(\xi_{g}\right)\left(\widetilde{O}_{1}\right)=O .
\end{aligned}
$$


So $h_{x_{0}}^{x_{0}}\left(\xi_{h}\right)$ maps $O$ to $O$, and by (8) it follows that the holonomy transport along $\xi_{g}$ defines an action of $H$ on $O$, which we further denote by

$$
h(g):=h_{x_{0}}^{x_{0}}\left(\xi_{g}\right): O \stackrel{\sim}{\rightarrow} O .
$$

Since $H$ is a finite group acting on $O$ with a fixed point $x_{0}$, by Bochner's Linearization Theorem, we can linearize the action around $x_{0}$. So, by shrinking $O$ if necessary, the action is isomorphic to the linear holonomy action of $H$ on $V$. In particular, this implies that $O$ contains arbitrarily small $H$-invariant open neighborhoods of $x_{0}$.

Since $\mathfrak{U}$ is a locally finite cover by relatively compact open sets, there are only finitely many chains in $\mathcal{Z}$ of a certain length. Denote by $\mathcal{Z}_{n}$ the set of chains of length at most $n$. Let $c \geq 1$ be such that $\xi_{g} \in \mathcal{Z}_{c}$ for all $g \in H$.

By the above, and by the basic properties of holonomy, there exist open neighborhoods $\left\{O_{n}\right\}_{n \geq 1}$ of $x_{0}$ in $O$ :

$$
\cdots \subset O_{n+1} \subset O_{n} \subset O_{n-1} \subset \cdots \subset O_{1} \subset O \subset V
$$

satisfying the following:

(1) for every chain $\xi \in \mathcal{Z}_{n}, O_{n} \subset O(\xi)$,

(2) for every two chains $\xi$, $\xi^{\prime} \in \mathcal{Z}_{n}$ and $x \in U_{k(\xi)} \cap U_{k\left(\xi^{\prime}\right)} \cap S$, such that the pairs $(\xi, x)$ and $\left(\xi^{\prime}, x\right)$ represent the same element in $\widetilde{S}$, we have that

$$
h_{x_{0}}^{x}(\xi)=h_{x_{0}}^{x}\left(\xi^{\prime}\right): O_{n} \hookrightarrow E_{x},
$$

(3) $O_{n}$ is $H$-invariant,

(4) for every $g \in H, \xi \in \mathcal{Z}_{n}$ and $x \in U_{k(\xi)} \cap S$, we have that

$$
h_{x_{0}}^{x}\left(\xi_{g} \cup \xi\right)=h_{x_{0}}^{x}(\xi) \circ h(g): O_{n+c} \hookrightarrow E_{x} .
$$

Denote by $\widetilde{S}_{n}$ the set of points in $\widetilde{x} \in \widetilde{S}$ for which every element in the orbit $\tilde{x} H$ can be represented by a pair $(\xi, x)$ with $\xi \in \mathcal{Z}_{n}$. Note that for $n \geq c, \widetilde{S}_{n}$ is nonempty, $H$-invariant, open, and connected. Consider the following $H$-invariant open neighborhood of $\widetilde{S} \times\left\{x_{0}\right\}$ :

$$
\mathcal{V}:=\bigcup_{n \geq c} \widetilde{S}_{n} \times O_{n+c} \subset \widetilde{S} \times V .
$$

On $\mathcal{V}$ we define the map

$$
\widetilde{\mathcal{H}}: \mathcal{V} \longrightarrow E, \quad \widetilde{\mathcal{H}}(\widetilde{x}, v):=h_{x_{0}}^{x}(\xi)(v)
$$

for $(\tilde{x}, v) \in \widetilde{S}_{n} \times O_{n+c}$, where $(\xi, x)$ is pair representing $\widetilde{x}$ with $\xi \in \mathcal{Z}_{n}$ and $x \in U_{k(\xi)}$. By the properties of the open sets $O_{n}, \widetilde{\mathcal{H}}$ is well defined. Since the holonomy transport is by germs of diffeomorphisms and preserves the foliation, it follows that $\widetilde{\mathcal{H}}$ is a foliated local diffeomorphism, which sends the trivial foliation on $\mathcal{V}$ with leaves $\mathcal{V} \cap(\widetilde{S} \times\{v\})$ to $\left.\mathcal{F}\right|_{E}$. 
We prove now that $\tilde{\mathcal{H}}$ is $H$-invariant. Let $(\widetilde{x}, v) \in \widetilde{S}_{n} \times O_{n+c}$ and $g \in H$. Consider chains $\xi$ and $\xi^{\prime}$ in $\mathcal{Z}_{n}$ representing $\tilde{x}$ and $\tilde{x} g$ respectively, with $x \in U_{k(\xi)} \cap U_{k\left(\xi^{\prime}\right)} \cap S$. Then $\xi^{\prime}$ and $\xi_{g} \cup \xi$ both belong to $\mathcal{Z}_{n+c}$ and $\left(\xi^{\prime}, x\right),\left(\xi_{g} \cup \xi, x\right)$ both represent $\tilde{x} g \in \widetilde{S}$. Using properties 2) and 4) of the open sets $O_{n}$, we obtain $H$-invariance:

$$
\begin{aligned}
\widetilde{\mathcal{H}}\left(\widetilde{x} g, h\left(g^{-1}\right) v\right) & =h_{x_{0}}^{x}\left(\xi^{\prime}\right)\left(h\left(g^{-1}\right) v\right)=h_{x_{0}}^{x}\left(\xi_{g} \cup \xi\right)\left(h\left(g^{-1}\right) v\right) \\
& =h_{x_{0}}^{x}(\xi) \circ h(g) \circ h\left(g^{-1}\right) v=h_{x_{0}}^{x}(\xi)(v)=\widetilde{\mathcal{H}}(\widetilde{x}, v) .
\end{aligned}
$$

Since the action of $H$ on $\mathcal{V}$ is free and preserves the foliation on $\mathcal{V}$, we obtain an induced local diffeomorphism of foliated manifolds:

$$
\mathcal{H}: \mathcal{V} / H \subset(\widetilde{S} \times V) / H \longrightarrow E
$$

We prove now that $\mathcal{H}$ is injective. Let $(\widetilde{x}, v),\left(\widetilde{x}^{\prime}, v^{\prime}\right) \in \mathcal{V}$ be such that

$$
\widetilde{\mathcal{H}}(\widetilde{x}, v)=\widetilde{\mathcal{H}}\left(\widetilde{x}^{\prime}, v^{\prime}\right)
$$

Denoting by $x=p(\widetilde{\mathcal{H}}(\widetilde{x}, v))=p\left(\widetilde{\mathcal{H}}\left(\widetilde{x}^{\prime}, v^{\prime}\right)\right)$, we have that $\widetilde{\mathcal{H}}(\widetilde{x}, v), \widetilde{\mathcal{H}}\left(\widetilde{x}^{\prime}, v^{\prime}\right) \in E_{x}$. Hence $\widetilde{x}$ and $\widetilde{x}^{\prime}$, both lie in the fiber of $\widetilde{S} \rightarrow S$ over $x$, thus there is a unique $g \in H$ with $\widetilde{x}^{\prime}=\tilde{x} g$. Let $n, m \geq c$ be such that $(\widetilde{x}, v) \in \widetilde{S}_{n} \times O_{n+c}$ and $\left(\widetilde{x}^{\prime}, v^{\prime}\right) \in \widetilde{S}_{m} \times O_{m+c}$, and assume also that $n \leq m$. Consider $\xi \in \mathcal{Z}_{n}$ and $\xi^{\prime} \in \mathcal{Z}_{m}$ such that $(\xi, x)$ represents $\tilde{x}$ and $\left(\xi^{\prime}, x\right)$ represents $\widetilde{x}^{\prime}$. Then we have that

$$
h_{x_{0}}^{x}(\xi)(v)=h_{x_{0}}^{x}\left(\xi^{\prime}\right)\left(v^{\prime}\right)
$$

Since both $\left(\xi^{\prime}, x\right)$ and $\left(\xi_{g} \cup \xi, x\right)$ represent $\widetilde{x}^{\prime} \in \widetilde{S}$, and both have length $\leq m+c$, again by the properties 2 ) and 4 ) we obtain

$$
h_{x_{0}}^{x}\left(\xi^{\prime}\right)\left(v^{\prime}\right)=h_{x_{0}}^{x}\left(\xi_{g} \cup \xi\right)\left(v^{\prime}\right)=h_{x_{0}}^{x}(\xi)\left(h(g)\left(v^{\prime}\right)\right) .
$$

Since $h_{x_{0}}^{x}(\xi)$ is injective, (9) implies that $v=h(g)\left(v^{\prime}\right)$. So, we obtain

$$
(\widetilde{x}, v)=\left(\widetilde{x}^{\prime} g^{-1}, h(g)\left(v^{\prime}\right)\right)
$$

which proves injectivity of $\mathcal{H}$.

\section{Thurston Stability around noncompact leaves}

To obtain the first order normal form result (Corollary 1), we will use the following extension to noncompact leaves of a result of Thurston [11].

Lemma 2 Let $S$ be an embedded leaf of a foliation such that $K_{\text {lin }}$, the kernel of $d h: \pi_{1}(S, x) \rightarrow H_{\text {lin }}$, is finitely generated and $H^{1}\left(\widetilde{S}_{\text {lin }}\right)=0$. Then the holonomy group $H_{\text {hol }}$ of $S$ coincides with the linear holonomy group $H_{\text {lin }}$ of $S$. 
Proof Denote by $V:=v_{x}$, the normal space at some $x \in S$. The linear holonomy gives an identification of the normal bundle of $S$ in $M$ with the vector bundle $\left(\widetilde{S}_{l i n} \times\right.$ $V) / H_{\text {lin }}$. Passing to a tubular neighborhood, we may assume that the foliation $\mathcal{F}$ is on $\left(\widetilde{S}_{l i n} \times V\right) / H_{l i n}$, and that its linear holonomy coincides with the holonomy of the flat bundle, i.e. the first order jet along $S$ of $\mathcal{F}$ equals the first order jet along $S$ of flat bundle foliation. Consider the covering map

$$
p: \widetilde{S}_{l i n} \times V \longrightarrow\left(\widetilde{S}_{l i n} \times V\right) / H_{l i n}
$$

The leaf $\widetilde{S}_{0}:=\widetilde{S}_{l i n} \times\{0\}$ of the pull-back foliation $p^{*}(\mathcal{F})$ on $\widetilde{S}_{l i n} \times V$ satisfies:

(1) $\widetilde{S}_{0}$ has trivial linear holonomy;

(2) $H^{1}\left(\widetilde{S}_{0}\right)=0$;

(3) $\pi_{1}\left(\widetilde{S}_{0}\right) \cong K_{\text {lin }}$ is finitely generated.

Thurston shows in [11] that, under the assumption that $\widetilde{S}_{0}$ is compact, the first two conditions imply that the holonomy group of $\widetilde{S}_{0}$ vanishes. It is straightforward to check that Thurston's argument actually doesn't use the compactness assumption, but it only uses condition (3); and we conclude that also in our case the holonomy at $\widetilde{S}_{0}$ of $p^{*}(\mathcal{F})$ vanishes.

Now consider a loop $\gamma$ in $S$ based at $x$ such that $[\gamma] \in K_{\text {lin }}$. This is equivalent to saying that $\gamma$ lifts to a loop in $\widetilde{S}_{\text {lin }}$, hence to a loop $\widetilde{\gamma}$ in $\widetilde{S}_{0}$. The holonomy transport along $\widetilde{\gamma}$ induced by $p^{*}(\mathcal{F})$ projects to the holonomy transport of $\gamma$ induced by $\mathcal{F}$, and since the first is trivial, so is the latter. This proves that $K_{l i n}$ is included in the kernel of $\pi_{1}(S, x) \rightarrow H_{h o l}$, and since the other inclusion always holds, we obtain that $H_{\text {hol }}=H_{\text {lin }}$.

\section{Foliated cohomology of products}

Let $M$ and $N$ be two manifolds. Consider the product foliation $T M \times N$ on $M \times N$, with leaves

$$
M \times\{y\} \subset M \times N, \quad y \in N .
$$

We denote the complex computing the corresponding foliated cohomology by

$$
\left(\Omega^{\bullet}(T M \times N), d\right) .
$$

The elements of $\Omega^{\bullet}(T M \times N)$ can be regarded as smooth families of forms on $M$ :

$$
\eta=\left\{\eta_{y} \in \Omega^{\bullet}(M)\right\}_{y \in N} \text { with } d \eta=\left\{d \eta_{y} \in \Omega^{\bullet+1}(M)\right\}_{y \in N} .
$$

Denote the corresponding cohomology groups by

$$
H^{\bullet}(T M \times N)
$$

We need two versions of these groups associated to a leaf $M \times\{x\}$, for a fixed $x \in N$. Denote the subcomplex of foliated forms vanishing on $M \times\{x\}$ by 


$$
\left(\Omega_{x}^{\bullet}(T M \times N), d\right),
$$

and the associated cohomology by

$$
H_{x}^{\bullet}(T M \times N)
$$

Finally, consider the complex of germs at $M \times\{x\}$ of foliated forms

$$
\left(\Omega_{\mathrm{g}_{x}}^{\bullet}(T M \times N), d\right) .
$$

This space is the quotient of $\Omega^{\bullet}(T M \times N)$ by the space of foliated forms that vanish on some open neighborhood of $M \times\{x\}$ in $M \times N$. The leafwise de Rham differential induces a differential on $\Omega_{\mathrm{g}_{x}}^{\bullet}(T M \times N)$. Denote the resulting cohomology by

$$
H_{\mathbf{g}_{x}}^{\bullet}(T M \times N) .
$$

Let $C_{x}^{\infty}(N)$ denote the space of smooth functions on $N$ vanishing at $x$, and let $C_{\mathrm{g}_{x}}^{\infty}(N)$ denote the space of germs of smooth functions on $N$ around $x$.

These three versions of foliated cohomology come with natural pairings with the homology of $M$, which yield maps:

$$
\begin{array}{r}
\Psi: H^{\bullet}(T M \times N) \longrightarrow \operatorname{Hom}\left(H_{\bullet}(M) ; C^{\infty}(N)\right), \\
\Psi_{x}: H_{x}^{\bullet}(T M \times N) \longrightarrow \operatorname{Hom}\left(H_{\bullet}(M) ; C_{x}^{\infty}(N)\right), \\
\Psi_{\mathrm{g}_{x}}: H_{\mathrm{g}_{x}}^{\bullet}(T M \times N) \longrightarrow \operatorname{Hom}\left(H_{\bullet}(M) ; C_{\mathrm{g}_{x}}^{\infty}(N)\right) .
\end{array}
$$

We explain the third map; the first two are constructed similarly. Consider an element $[\eta] \in H_{\mathrm{g}_{x}}^{q}(T M \times N)$, which is represented by a foliated $q$-form $\eta$ which is closed on some open set containing $M \times\{x\}$. The corresponding linear map:

$$
\Psi_{\mathrm{g}_{x}}([\eta]): H_{q}(M) \longrightarrow C_{\mathrm{g}_{x}}^{\infty}(N)
$$

is constructed as follows. Represent an element $[c] \in H_{q}(M)$ as $c=\sum_{i} a_{i} \sigma_{i}$, where $\sigma_{i}: \Delta_{q} \rightarrow M$ are smooth $q$-simplices. Define

$$
\langle\eta, c\rangle \in C^{\infty}(N), \quad y \mapsto \sum_{i} a_{i} \int_{\Delta_{q}}\left(\sigma_{i} \times\{y\}\right)^{*}(\eta) .
$$

The germ at $x$ of the function $\langle\eta, c\rangle$ is independent of the choice of the representatives, yielding a well-defined element $\Psi_{\mathrm{g}_{x}}([\eta])([c]):=\langle[\eta],[c]\rangle \in C_{\mathrm{g}_{x}}^{\infty}(N)$.

Lemma 3 The maps from (10) are linear isomorphisms.

Proof Denote the constant sheaves on $M$ associated to the groups $C^{\infty}(N), C_{x}^{\infty}(N)$ and $C_{\mathrm{g}_{x}}^{\infty}(N)$ by $\mathcal{S}_{1}, \mathcal{S}_{2}$ and $\mathcal{S}_{3}$, respectively. By standard arguments, the de Rham differential along $M$ induces resolutions $\mathcal{S}_{i} \rightarrow \mathcal{C}_{i}^{\bullet}$ by fine sheaves on $M$ :

$$
\mathcal{C}_{1}^{\bullet}(U):=\Omega^{\bullet}(T U \times N), \quad \mathcal{C}_{2}^{\bullet}(U):=\Omega_{x}^{\bullet}(T U \times N), \quad \mathcal{C}_{3}^{\bullet}(U):=\Omega_{\mathrm{g}_{x}}^{\bullet}(T U \times N) .
$$


Hence, the foliated cohomologies from (10) are isomorphic to the sheaf cohomologies with coefficients in $\mathcal{S}_{1}, \mathcal{S}_{2}$ and $\mathcal{S}_{3}$ respectively. On the other hand, for any vector space $V$, denoting by $\underline{V}$ the constant sheaf on $M$, one has a natural isomorphism:

$$
\Phi_{V}: H^{\bullet}(M ; \underline{V}) \stackrel{\sim}{\rightarrow} \operatorname{Hom}\left(H_{\bullet}(M) ; V\right)
$$

Hence, we obtain isomorphisms:

$$
\begin{aligned}
& \Phi: H^{\bullet}(T M \times N) \stackrel{\sim}{\rightarrow} \operatorname{Hom}\left(H_{\bullet}(M) ; C^{\infty}(N)\right), \\
& \Phi_{x}: H_{x}^{\bullet}(T M \times N) \stackrel{\sim}{\rightarrow} \operatorname{Hom}\left(H_{\bullet}(M) ; C_{x}^{\infty}(N)\right), \\
& \Phi_{\mathrm{g}_{x}}: H_{\mathrm{g}_{x}}^{\bullet}(T M \times N) \stackrel{\sim}{\rightarrow} \operatorname{Hom}\left(H_{\bullet}(M) ; C_{\mathrm{g}_{x}}^{\infty}(N)\right) .
\end{aligned}
$$

We still have to check that these maps coincide with those from (10). For this we will exploit the naturality of the maps in (11).

In the first case, consider the evaluation map ev $v_{y}: C^{\infty}(N) \rightarrow \mathbb{R}$, for $y \in N$. This induces a sheaf map $e v_{y}^{M}: \mathcal{S}_{1} \rightarrow \underline{\mathbb{R}}$ into the constant sheaf over $M$, which is covered by a map $e v_{y}^{M}: \mathcal{C}_{1}^{\bullet} \rightarrow \Omega_{M}^{\bullet}$ into the standard de Rham resolution of $\underline{\mathbb{R}}$. Hence the map $H^{\bullet}\left(M ; \mathcal{S}_{1}\right) \rightarrow H^{\bullet}(M ; \underline{\mathbb{R}})$ induced by $e v_{y}$ becomes

$$
H^{\bullet}(T M \times N) \stackrel{e v_{y}^{M}}{\longrightarrow} H^{\bullet}(M), \quad[\omega] \mapsto\left[\left.\omega\right|_{M \times\{y\}}\right] .
$$

By naturality of (11), it follows that the following square commutes:

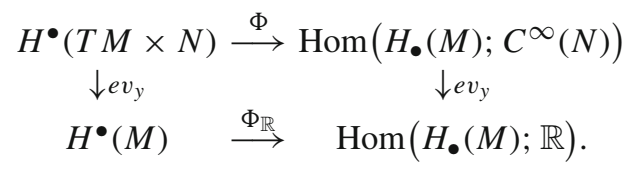

Since $\Phi_{\mathbb{R}}$ is the usual isomorphism given by integration, and by the explicit description of the map $\Psi$, this implies that $\Psi=\Phi$.

For the second map in (10) and (12) we proceed similarly, but using the inclusion $i: C_{x}^{\infty}(N) \rightarrow C^{\infty}(N)$ instead of $e v_{y}$. This gives rise to a sheaf map $\mathcal{S}_{2} \rightarrow \mathcal{S}_{1}$ which lifts to their resolutions, and then we obtain a commutative square

$$
\begin{gathered}
H_{x}^{\bullet}(T M \times N) \stackrel{\Phi_{x}}{\longrightarrow} \operatorname{Hom}\left(H_{\bullet}(M) ; C_{x}^{\infty}(N)\right) \\
\downarrow_{i} \\
H^{\bullet}(T M \times N) \stackrel{\Phi}{\longrightarrow} \operatorname{Hom}\left(H_{\bullet}(M) ; C^{\infty}(N)\right) .
\end{gathered}
$$

Using also that $\Psi=\Phi$, this implies the equality $\Psi_{x}=\Phi_{x}$.

Similarly, for the third map in (10) and (12), but using the projection map $p$ : $C^{\infty}(N) \rightarrow C_{\mathrm{g}_{x}}^{\infty}(N)$ (instead of the inclusion), we obtain a commutative square 


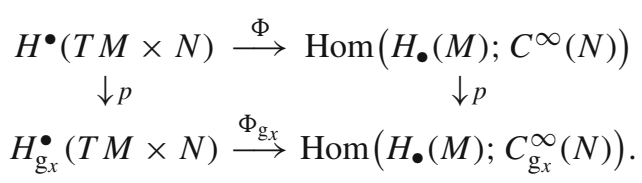

Again, since $\Psi=\Phi$, we obtain that $\Psi_{\mathrm{g}_{x}}=\Phi_{\mathrm{g}_{x}}$. This concludes the proof.

We will use the following consequences of Lemma 3 (the first appeared in [5]).

Corollary 2 Let $\eta \in \Omega^{q}(T M \times N)$ be a foliated $q$-form such that $\eta_{y} \in \Omega^{q}(M)$ $i$ exact for all $y \in N$. Then there exists $\theta \in \Omega^{q-1}(T M \times N)$ such that $d \theta=\eta$. Moreover, if $\eta_{x}=0$ for some $x \in N$, then one can choose $\theta$ such that $\theta_{x}=0$.

Proof In the first case, we need that $[\eta]=0$ in $H^{\bullet}(T M \times N)$, and in the second, that $[\eta]=0$ in $H_{x}^{\bullet}(T M \times N)$. Since $\left\langle\left[\eta_{y}\right],[c]\right\rangle=0$, for all $[c] \in H_{q}(M)$ and all $y \in N$, the description of the maps $\Psi$ and $\Psi_{x}$ and Lemma 3 imply the result.

Corollary 3 Let $\eta$ be a closed foliated $q$-form defined on some open neighborhood $\mathcal{U} \subset M \times N$ of $M \times\{x\}$. Then there exists a closed foliated $q$-form $\tilde{\eta}$ on $M \times N$, such that $\left.\eta\right|_{\tilde{\mathcal{U}}}=\left.\tilde{\eta}\right|_{\tilde{\mathcal{U}}}$, for some open set $\widetilde{\mathcal{U}} \subset \mathcal{U}$ containing $M \times\{x\}$.

Proof First, we claim that the projection $p: \Omega^{\bullet}(T M \times N) \rightarrow \Omega_{\mathrm{g}_{x}}^{\bullet}(T M \times N)$ induces a surjective map in cohomology. By the description of the maps $\Psi$ and $\Psi_{\mathrm{g}_{x}}$, we have a commutative diagram

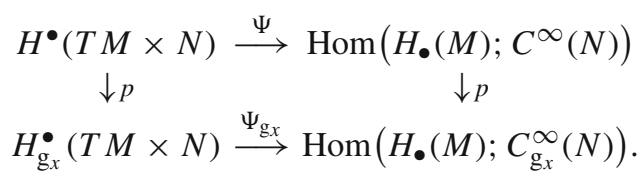

By Lemma 3, the horizontal maps are isomorphisms, and since the vertical map on the right is surjective, so is the vertical map on the left.

Consider a foliated $q$-form $\eta^{\prime} \in \Omega^{q}(T M \times N)$, such that $\eta^{\prime}\left|\mathcal{U}^{\prime}=\eta\right|_{\mathcal{U}^{\prime}}$ for some open neighborhood $\mathcal{U}^{\prime} \subset \mathcal{U}$ of $M \times\{x\}$. Then $\eta^{\prime}$ defines a class $\left[\eta^{\prime}\right] \in H_{\mathrm{g}_{x}}^{q}(T M \times N)$. By the above, there is a closed foliated $q$-form $\eta^{\prime \prime} \in \Omega^{q}(T M \times N)$, such that $\left[\eta^{\prime \prime}\right]=\left[\eta^{\prime}\right] \in$ $H_{\mathrm{g}_{x}}^{q}(T M \times N)$. Thus, there is some foliated $q-1$-form $\theta$ and an open neighborhood $\tilde{\mathcal{U}} \subset \mathcal{U}^{\prime}$ of $M \times\{x\}$ such that $\left.\eta^{\prime}\right|_{\tilde{\mathcal{U}}}=\left.\left(\eta^{\prime \prime}+d \theta\right)\right|_{\tilde{\mathcal{U}}}$. The closed foliated $q$-form $\widetilde{\eta}:=\eta^{\prime \prime}+d \theta$ satisfies the conclusion: $\left.\tilde{\eta}\right|_{\tilde{\mathcal{U}}}=\left.\eta\right|_{\tilde{\mathcal{U}}}$.

\section{Equivariant submersions}

We prove that submersions can be equivariantly linearized.

Lemma 4 Let $G$ be compact Lie group acting linearly on the vector spaces $V$ and $W$. Consider $f: V \rightarrow W$ a smooth $G$-equivariant map, such that $f(0)=0$. If $f$ is a submersion at 0 , then there exists a $G$-equivariant embedding $\chi: U \hookrightarrow V$, where $U$ is an invariant open around 0 in $V$, such that $\chi(0)=0$ and

$$
f(\chi(v))=d f_{0}(v), \text { for } v \in U
$$


Proof Since $G$ is compact, we can find a $G$-equivariant projection $p_{K}: V \rightarrow K$, where $K:=\operatorname{ker}\left(d f_{0}\right)$. The differential at 0 of the $G$-equivariant map

$$
\left(f, p_{K}\right): V \longrightarrow W \times K, v \mapsto\left(f(v), p_{K}(v)\right)
$$

is $\left(d f_{0}, p_{K}\right)$. So $\left(f, p_{K}\right)$ is a diffeomorphism when restricted to some open $U_{0}$ in $V$ around 0 , which we may assume to be $G$-invariant. Define the embedding as follows

$$
\chi: U \hookrightarrow V, \quad \chi:=\left(f, p_{K}\right)^{-1} \circ\left(d f_{0}, p_{K}\right),
$$

where $U:=\left(d f_{0}, p_{K}\right)^{-1}\left(U_{0}\right)$. Clearly $U$ is $G$-invariant, $\chi$ is $G$-equivariant and $\chi(0)=0$. Since

$$
\left(f(\chi(v)), p_{K}(\chi(v))\right)=\left(d f_{0}(v), p_{K}(v)\right)
$$

we also have that $f(\chi(v))=d f_{0}(v)$.

\section{The Moser Lemma for symplectic foliations}

The following is a version for symplectic foliations of the Moser Lemma.

Lemma 5 Let $(M, \mathcal{F}, \omega)$ be a symplectic foliation. Consider a foliated 1-form

$$
\alpha \in \Omega^{1}(T \mathcal{F})
$$

that vanishes on an embedded saturated submanifold $Z$ of $M$. Then $\omega+d_{\mathcal{F}} \alpha$ is nondegenerate in a neighborhood $U$ of $Z$, and the resulting symplectic foliation

$$
\left(U,\left.\mathcal{F}\right|_{U},\left.\omega\right|_{U}+\left.d_{\mathcal{F}} \alpha\right|_{U}\right)
$$

is isomorphic around $Z$ to $(M, \mathcal{F}, \omega)$ by a foliated diffeomorphism that fixes $Z$.

Proof Since $\alpha$ vanishes on $Z$ and $Z$ is saturated, it follows that also $d_{\mathcal{F}} \alpha$ vanishes on $Z$. Thus, there is an open $V$ around $Z$ such that $\omega+d_{\mathcal{F}} \alpha$ is nondegenerate along the leaves of $\left.\mathcal{F}\right|_{V}$. Moreover, by the classical tube lemma from topology, we may choose $V$ such that

$$
\omega_{t}:=\omega+t d_{\mathcal{F}} \alpha \in \Omega^{2}(T \mathcal{F})
$$

is nondegenerate along the leaves of $\left.\mathcal{F}\right|_{V}$, for all $t \in[0,1]$. Consider the time dependent vector field $X_{t}$ on $V$, tangent to $\mathcal{F}$, determined by

$$
\iota_{X_{t}} \omega_{t}=-\alpha, \quad X_{t} \in \Gamma\left(\left.T \mathcal{F}\right|_{V}\right)
$$

Since $X_{t}$ vanishes along $Z$, again by the tube lemma, there is an open $O \subset V$ around $Z$, such that the flow $\Phi_{X}^{t}$ of $X_{t}$ is defined up to time 1 on $O$. We claim that $\Phi_{X}^{1}$ gives 
the desired isomorphism. Clearly $\Phi_{X}^{1}$ preserves the foliation and is the identity on $Z$. On each leaf $S$, we have that

$$
\frac{d}{d t} \Phi_{X}^{t *}\left(\left.\omega_{t}\right|_{S}\right)=\Phi_{X}^{t *}\left(\left.L_{X_{t}} \omega_{t}\right|_{S}+\left.d_{\mathcal{F}} \alpha\right|_{S}\right)=\Phi_{X}^{t *}\left(\left.d \iota_{X_{t}} \omega_{t}\right|_{S}+\left.d \alpha\right|_{S}\right)=0
$$

Thus $\Phi_{X}^{t *}\left(\left.\omega_{t}\right|_{S}\right)$ is constant, and since $\Phi_{X}^{0}=\mathrm{Id}$, we have that

$$
\Phi_{X}^{1 *}\left(\left.\left(\omega+d_{\mathcal{F}} \alpha\right)\right|_{S}\right)=\left.\omega\right|_{S}
$$

So, $\Phi_{X}^{1}$ is an isomorphism between the symplectic foliations

$$
\Phi_{X}^{1}:\left(O,\left.\mathcal{F}\right|_{O},\left.\omega\right|_{O}\right) \stackrel{\sim}{\rightarrow}\left(U,\left.\mathcal{F}\right|_{U},\left.\omega\right|_{U}+\left.d_{\mathcal{F}} \alpha\right|_{U}\right)
$$

where $U:=\Phi_{X}^{1}(O)$.

We conclude this section with:

Proof of Proposition 1 A second 2-form is of the form

$$
\Omega^{\prime}=\Omega+d_{\nabla} \lambda
$$

for some $\lambda \in \Omega^{1}\left(T \mathcal{F} ; v^{*}\right)$. We apply the Lemma 5 to the symplectic foliation

$$
\left(\nu, \mathcal{F}_{\nu}, p^{*}(\omega)+\delta \omega\right)
$$

and the foliated 1-form $\alpha:=\mathcal{J}(\lambda)$ which vanishes along $M$. The resulting diffeomorphism is foliated. In particular, above any leaf $S$ of $\mathcal{F}$, the diffeomorphism sends the local model corresponding to $\Omega$ to the local model corresponding to $\Omega^{\prime}$.

\section{Proof of Theorem 1}

Since the holonomy of $S$ is finite, it coincides with the linear holonomy. Consider $x \in S$ and denote by $V:=v_{x}$, by $H:=H_{\text {hol }}=H_{\text {lin }}$, and by $\widetilde{S}:=\widetilde{S}_{\text {hol }}=\widetilde{S}_{\text {lin }}$. Applying Lemma 1, we obtain that some neighborhood of $S$ in $M$ is diffeomorphic as a foliated manifold to an open $\mathcal{U}$ around $S$ in the flat bundle

$$
(\widetilde{S} \times V) / H
$$

The symplectic leaves correspond to the connected components of $S_{v} \cap \mathcal{U}$, where

$$
S_{v}:=(\widetilde{S} \times H v) / H, \quad v \in V .
$$


We claim that there exists $\omega_{1}$, a closed foliated 2-form on $(\widetilde{S} \times V) / H$ that extends $\left.\omega\right|_{\mathcal{U}_{1}}$, for some open $\mathcal{U}_{1} \subset \mathcal{U}$ around $S$. For this, consider the projection

$$
p: \widetilde{S} \times V \longrightarrow(\widetilde{S} \times V) / H,
$$

and denote by $\widetilde{\mathcal{U}}:=p^{-1}(\mathcal{U})$ and by $\widetilde{\omega}:=p^{*}(\omega)$, which is a closed foliated 2-form on the product foliation restricted to $\widetilde{\mathcal{U}}$. By Corollary 3 , there is a closed extension $\widetilde{\omega}_{0}$ of $\widetilde{\omega} \mid \widetilde{\mathcal{U}}_{0}$, where $\widetilde{\mathcal{U}}_{0} \subset \widetilde{\mathcal{U}}$ is an open around $\widetilde{S} \times\{0\}$. Define $\widetilde{\omega}_{1}$ by averaging over $H$

$$
\widetilde{\omega}_{1}:=\frac{1}{|H|} \sum_{g \in H} g^{*}\left(\widetilde{\omega}_{0}\right) .
$$

Since $\widetilde{\omega}$ is invariant, it follows that $\widetilde{\omega}_{1}$ coincides with $\widetilde{\omega}$ on $\widetilde{\mathcal{U}}_{1}:=\bigcap_{g \in H} g \widetilde{\mathcal{U}}_{0}$. Since $\widetilde{\omega}_{1}$ is invariant, it is of the form $\widetilde{\omega}_{1}=p^{*}\left(\omega_{1}\right)$, where $\omega_{1}$ is a closed foliated 2 -form on $(\widetilde{S} \times V) / H$, which extends the restriction to $\mathcal{U}_{1}:=p\left(\widetilde{\mathcal{U}}_{1}\right)$ of $\omega$.

We will identify foliated $q$-forms $\eta$ on $(\widetilde{S} \times V) / H$, with smooth $H$-equivariant families $\left\{\eta_{v} \in \Omega^{q}(\widetilde{S})\right\}_{v \in V}$, where $\eta_{v}:=\left.p^{*}(\eta)\right|_{\tilde{S} \times\{v\}}$.

We compute now the variation of $\omega$ at $S$. Since $\omega$ and $\omega_{1}$ coincide around $S$, they have the same variation at $S$. Using the extension of $\omega$ (or equivalently of $\omega_{1}$ ) that vanishes on vectors tangent to the fibers of the projection to $S$, we see that the variation $\delta_{S} \omega$ is given by the $H$-equivariant family:

$$
\delta_{S} \omega_{v}:=\left.\frac{d}{d \epsilon} \omega_{\epsilon v}\right|_{\epsilon=0} \in \Omega^{2}(\widetilde{S}),
$$

The local model is represented by the $H$-equivariant family of 2-forms:

$$
j_{S}^{1}(\omega)_{v}=p^{*}\left(\omega_{S}\right)+\delta_{S} \omega_{v} \in \Omega^{2}(\widetilde{S})
$$

Consider the $H$-equivariant map

$$
f: V \longrightarrow H^{2}(\widetilde{S}), \quad f(v)=\left[\omega_{1, v}\right]-\left[p^{*}\left(\omega_{S}\right)\right]
$$

Smoothness of $f$ follows from Lemma 3. Clearly, $f(0)=0$ and its differential at 0 is the cohomological variation

$$
d f_{0}(v)=\left[\delta_{S} \omega\right] v, \quad \forall v \in V
$$

By our hypothesis, $f$ is a submersion at 0 . So we can apply Lemma 4 to find an $H$-equivariant embedding

$$
\chi: U \hookrightarrow V
$$

where $U$ is an $H$-invariant open neighborhoods of 0 in $V$, such that

$$
\chi(0)=0 \text { and } f(\chi(v))=d f_{0}(v) .
$$


By $H$-equivariance, $\chi$ induces a foliation preserving embedding

$$
\widetilde{\chi}:(\widetilde{S} \times U) / H \hookrightarrow(\widetilde{S} \times V) / H, \quad \widetilde{\chi}([y, v])=[y, \chi(v)]
$$

that restricts to a diffeomorphism between the leaf $S_{v}$ and the leaf $S_{\chi(v)}$. The pullback of $\omega_{1}$ under $\tilde{\chi}$ is the $H$-equivariant family

$$
\omega_{2}=\left\{\omega_{2, v}:=\omega_{1, \chi(v)}\right\}_{v \in U}
$$

We have that

$$
\left[\omega_{2, v}\right]-\left[p^{*}\left(\omega_{S}\right)\right]=\left[\omega_{1, \chi(v)}\right]-\left[p^{*}\left(\omega_{S}\right)\right]=f(\chi(v))=d f_{0}(v)=\left[\delta_{S} \omega\right] v
$$

Equivalently, this relation can be rewritten as

$$
\omega_{2, v}=j_{S}^{1}(\omega)_{v}+\alpha_{v}, \forall v \in U
$$

where $\left\{\alpha_{v}\right\}_{v \in U}$ is an $H$-equivariant family of exact 2-forms that vanishes for $v=0$. By Corollary $2, p^{*}(\alpha)$ is an exact foliated form on $\widetilde{S} \times U$, and moreover, we can choose a primitive $\widetilde{\beta} \in \Omega^{1}(T \widetilde{S} \times U)$ such that $\widetilde{\beta}_{0}=0$. By averaging, we may also assume that $\widetilde{\beta}$ is $H$-equivariant, thus it is of the form $\widetilde{\beta}=p^{*}(\beta)$ for a foliated 1 -form on $\beta$ on $(\widetilde{S} \times U) / H$ that vanishes along $S$. We obtain:

$$
\omega_{2}=j_{S}^{1} \omega+d \beta
$$

Applying Lemma 5, we conclude that, on some open around $S, j_{S}^{1} \omega$ and $\omega_{2}$ are related by a foliated diffeomorphism. Now, $\omega_{2}$ and $\omega_{1}$ are related by $\widetilde{\chi}$, and $\omega_{1}$ and $\omega$ have the same germ around $S$. This concludes the proof.

\section{Proof of Corollary 1}

Schreier's Lemma says that a subgroup of finite index of a finitely generated group is also finitely generated (see e.g. Sect. 5.6 in [10]). Hence, $K_{\text {lin }}$ is finitely generated. By Lemma 2, $H_{h o l}=H_{l i n}$, in particular $H_{h o l}$ is finite, and so we are in the setting of Theorem 1.

\section{Some global considerations}

A natural question, which was proposed by the referee, is that of giving a version of the global Reeb stability for symplectic foliations. In this last section, we discuss an interesting aspect of this problem, namely, that the appropriate compactness conditions are incompatible with the surjectivity of the cohomological variation. The argument used is similar to the one in Sect. 2.1 of [8].

We consider a symplectic foliation $(M, \mathcal{F}, \omega)$, and we assume that all leaves of $\mathcal{F}$ are compact with finite holonomy group; in particular, this setting includes the 
conclusion of the classical global Reeb stability. This allows us to define a smooth volume map on $M$, which is constant on the leaves:

$$
\text { vh }: M \longrightarrow(0, \infty), \quad \operatorname{vh}(x):=\left|H_{x}\right| \int_{S_{x}} \omega_{S_{x}}^{k},
$$

where $H_{x}$ is the holonomy group of the leaf $\left(S_{x}, \omega_{S_{x}}\right)$ through $x$. To see that this map is indeed smooth, write locally the foliation as the flat bundle $\left(\widetilde{S}_{x} \times V\right) / H_{x}$, and represent $\omega$ by an $H_{x}$-equivariant smooth family of 2-forms $\left\{\omega_{v} \in \Omega^{2}\left(\widetilde{S}_{x}\right)\right\}_{v \in V}$. The pullback of the map vh to $\widetilde{S}_{x} \times V$ is simply the volume map $(y, v) \mapsto \int_{\widetilde{S}_{x}} \omega_{v}^{k}$, which is clearly smooth. Next, we claim that if the cohomological variation is onto, then vh is submersion. In the local model, the pullback to $\widetilde{S}_{x} \times V$ of the map vh is decomposable as

$$
V \stackrel{v \mapsto\left[\omega_{v}\right]}{\longrightarrow} H^{2}\left(\widetilde{S}_{x}\right) \stackrel{[\eta] \mapsto[\eta]^{k}}{\longrightarrow} H^{2 k}\left(\widetilde{S}_{x}\right) \stackrel{\int_{\widetilde{S}_{x}}}{\longrightarrow} \mathbb{R} .
$$

The derivative of the first map is the cohomological variation, which is surjective; at $\eta:=\omega_{0}$, since $[\eta]^{k} \neq 0$, the derivative in the direction of $[\eta]$ is nonzero, thus it is surjective; and finally, the last map is an isomorphism. This argument, implies that vh is a submersion. In particular, we conclude that $M$ cannot be compact.

Acknowledgments This research was financially supported by the ERC Starting Grant no. 279729.

Open Access This article is distributed under the terms of the Creative Commons Attribution License which permits any use, distribution, and reproduction in any medium, provided the original author(s) and the source are credited.

\section{References}

1. Cantwell, J., Conlon, L.: Reeb stability for noncompact leaves in foliated 3-manifolds. Proc. Am. Math. Soc. 83(2), 408-410 (1981)

2. Crainic, M., Fernandes, R.L.: Integrability of poisson brackets. J. Differ. Geom. 66, 71-137 (2004)

3. Crainic, M., Fernandes, R.L.: Stability of symplectic leaves. Inven. Math. 180(3), 481-533 (2010)

4. Crainic, M., Mărcuţ, I.: A normal form theorem around symplectic leaves. J. Differ. Geom. 92(3), 417-461 (2012)

5. Gotay, M.J., Lashof, R., Śniatycki, J., Weinstein, A.: Closed forms on symplectic fibre bundles. Commentarii Mathematici Helvetici 58(1), 617-621 (1983)

6. Inaba, T.: Reeb stability for noncompact leaves. Topology 22(1), 105-118 (1983)

7. Mărcuţ, I.: Normal forms in Poisson geometry, PhD thesis, Utrecht University (2013). arXiv:1301.4571

8. Mărcuţ, I.: Rigidity around poisson submanifolds. Acta Math. 213(1), 137-198 (2014)

9. Moerdijk, I., Mrčun, J.: Introduction to Foliations and Lie groupoids, Cambridge Studies in Advanced Mathematics, 91. Cambridge University Press, Cambridge (2003)

10. Rotman, J.: Advanced Modern Algebra. Prentice Hall Inc, Upper Saddle River, NJ (2002)

11. Thurston, W.: A generalization of the Reeb stability theorem. Topology 13, 347-352 (1974)

12. Vorobjev, Y.: Coupling tensors and poisson geometry near a single symplectic leaf. Banach Cent. Publ. 54, 249-274 (2001) 